\title{
POSITIVE SOLUTIONS FOR THE 1-DIMENSIONAL \\ GENERALIZED $p$-LAPLACIAN INVOLVING A REAL PARAMETER *
}

\author{
EDSON ARRÁZOLA \\ IMECC-UNICAMP, Brasil \\ and \\ PEDRO UBILLA \\ Universidad de Santiago de Chile, Chile
}

\begin{abstract}
In this paper we study existence and multiplicity of positive solutions of the Dirichlet problem
\end{abstract}

KEY WORDS AND PHRASES: positive solution, strongly nonlinear, superlinear and sublinear problem.

*The authors are supported by grants DICYT 04-9533 UL from Universidad de Santiago de Chile and FONDECYT 1950605-95 


\section{Introduction}

In this paper we study positive solutions of the Dirichlet problem

$$
(P)_{\lambda} \quad\left\{\begin{array}{c}
-\left(\varphi\left(u^{\prime}\right)\right)^{\prime}=\lambda f(u) \\
u(0)=u(1)=0,
\end{array} \quad \text { in } \quad(0,1)\right.
$$

where $\lambda \in \mathbb{R}, \quad \varphi: \mathbb{R} \rightarrow \mathbb{R}$ is an increasing odd homeomorphism and $f: \mathbb{R} \rightarrow \mathbb{R}$ is continuous. By a solution of problem $(P)_{\lambda}$ we will understand a function $u \in C^{1}(0,1)$ such that $\varphi\left(u^{\prime}\right) \in C^{1}(0,1)$ satisfying the equations in $(P)_{\lambda}$.

For the partial case, the existence of positive solutions for the problem $-\Delta u=\lambda f(u)$ in $\Omega, u=0$ on $\partial \Omega$, has been widely studied by many authors (see De Figueiredo, Lions and Nussbaum [2], Brezis and Turner [1], among others). An informative survey concerning this problem can be founded in the paper of Lions [6], where the author consider different behaviors of $f$ in order to obtain the existence and multiplicity results of positive solutions. On the other hand, taking into account that $f$ is superlinear and the partial differential operator is asymptotic to a power at zero and at infinity, Narukawa and Suzuky [7] studied the existence of positive solutions, and obtained the existence of a $\lambda^{*}>0$, such that for any $0<\lambda<\lambda^{*}$ the described problem has at least two positive solutions, neverthless the existence of positive solutions for $\lambda \geq \lambda^{*}$ was not considered. In the 1-dimensional case is also possible to obtain existence results for $\lambda \geq \lambda^{*}$, thus for an operator satisfying the conditions $\left(A_{0}\right),\left(A_{\infty}\right)$ and for the non-linearity satisfying appropiate jumping conditions (sce (1.2)) we study the existence of positive solutions. In particular, in our main result (Theorem 3.2), besides proving that $(P)_{\lambda}$ has at least two positive solutions for any $0<\lambda<\lambda^{*}$, we prove that it has at least one positive solution for $\lambda=\lambda^{*}$, and has no positive solution for any $\lambda>\lambda^{*}$. To this goal, first we construct the solutions of $(P)_{\lambda}$ using the Shooting Method (see Ubilla [10], and Guedda and Veron [5]), and briefly review and refine some results finded in [4] to obtain Lemma 2.1. Then we formulate and prove our results, and finally we present some examples.

Suppose that the function $\varphi$ satisfies the next conditions,

$\left(A_{0}\right) \quad \lim _{x \rightarrow 0} \frac{\varphi(\sigma x)}{\varphi(x)}=\sigma^{q-1}$ for some $q>1$ and for all $\sigma \in(0,1)$,

$\left(A_{\infty}\right) \quad \lim _{x \rightarrow+\infty} \frac{\varphi(\sigma x)}{\varphi(x)}=\sigma^{p-1}$ for some $p>1$ and for all $\sigma \in(0,1)$, 
that is, $\varphi$ is a regularly varying function of positive index at zero and at infinity. In general this kind of functions are used in Probability: references may be found in Resnick [8], and Seneta [9]. Nevertheless, in the context that we will use here, these functions were considered recently in [3] and [4].

We also assume that $f$ is an odd function which satisfies

$$
x f(x)>0 \quad \forall x \neq 0
$$

$$
\lim _{x \rightarrow 0} \frac{f(x)}{\varphi(x)}=a \quad \text { and } \quad \lim _{x \rightarrow+\infty} \frac{f(x)}{\varphi(x)}=A,
$$

where $a, A \in[0,+\infty]$.

Finally we introduce some notation to be used in the subsequent sections. Let us set

$$
\Phi(x)=: \int_{0}^{x} \varphi(s) d s, \quad \Phi_{*}(x)=: \int_{0}^{x} \varphi^{-1}(s) d s \quad \text { and } \quad F(x)=: \int_{0}^{x} f(t) d t .
$$

Since $\varphi$ and $\varphi^{-1}$ are strictly increasing odd functions, $\Phi$ and $\Phi_{*}$ are strictly increasing even functions on $(0,+\infty)$. Then, the inverse of $\Phi_{*} \mid(0,+\infty)$ is well-defined and we will write $\Phi_{*}^{-1}$. Finally, we denote by $\lambda_{k}(p)$ the $k$-th eigenvalue of the problem

$$
\left\{\begin{array}{c}
-\left(\left|u^{\prime}\right|^{p-2} u^{\prime}\right)^{\prime}=\lambda|u|^{p-2} u, \\
u(0)=u(1)=0,
\end{array}\right.
$$

where $p>1$. One can prove that

$$
\lambda_{k}(p)=k^{p} \lambda_{1}(p)=k^{p}(p-1)\left[2 \int_{0}^{1} \frac{d z}{\left(1-z^{p}\right)^{1 / p}}\right]^{p},
$$

using the ideas finded in [5] and [10].

\section{Construction of the Solutions}

We observe that if problem $(P)_{\lambda}$ has non-trivial solutions, then $\lambda>0$. In fact, let $u$ a solution of $(P)_{\lambda}$, then multiplying by $u$ and integrating by parts over $(0,1)$, yields

$$
\int_{0}^{1} u^{\prime} \varphi\left(u^{\prime}\right) d t=\lambda \int_{0}^{1} u f(u) d t
$$


Hence using (1.1) and the fact that $\varphi$ is an increasing odd function we obtain $\lambda>0$.

Now, we consider the initial value problem $(I)$ associated to $(P)_{\lambda}$

$$
(I) \quad\left\{\begin{array}{c}
-\left(\varphi\left(u^{\prime}\right)\right)^{\prime}=\lambda f(u) \\
u(0)=0 \\
u^{\prime}(0)=\alpha>0 .
\end{array}\right.
$$

Let $u$ a solution of $(I)$, then multiplying $(I)$ by $u^{\prime}$ and intcgrating we obtain

$$
\left(\Phi_{*} \circ \varphi\right)\left(u^{\prime}(t)\right)+\lambda F(u(t))=C=\text { constant },
$$

so, taking $W=: \Phi_{*} \circ \varphi$, yields

$$
W\left(u^{\prime}(t)\right)+\lambda F(u(t))=C .
$$

Setting $t=0$ in (2.1), we obtain $C=W(\alpha)$, thus the energy relation for $(I)$ is

$$
W\left(u^{\prime}(t)\right)=W(\alpha)-\lambda F(u(t)) .
$$

We will denote by $S(\alpha, \lambda)$ the unique positive zero of the function $W(\alpha)-$ $\lambda F(\cdot)$, then

$$
F(S(\alpha, \lambda))=\frac{1}{\lambda} W(\alpha)
$$

Thus we observe that $S(\alpha, \lambda) \rightarrow+\infty$ as $\alpha \rightarrow+\infty$ for each $\lambda>0$.

Using similar arguments as in [4] and [10], we can prove the existence of solutions $\{-u, u\}$ for the problen $(I)$ such that

$$
u(2 k \theta(\alpha, \lambda))=0 \quad, \quad k=0, \pm 1, \pm 2, \ldots,
$$

where

$$
\theta(\alpha, \lambda)=\int_{0}^{S(\alpha, \lambda)} \frac{d t}{W_{+}^{-1}(W(\alpha)-\lambda F(t))}, \quad \text { with } \quad W_{+}=\left.W\right|_{[0,+\infty)} .
$$

Therefore, the only zeros of the solutions of problem $(I)$ are $\{2 k \theta(\alpha, \lambda) \mid k \in \mathbb{Z}\}$, moreover, they are $4 \theta(\alpha, \lambda)$ - periodic.

Now, we observe that if there exist positive constants $\alpha$ and $\lambda$ such that 


$$
\frac{1}{2 \theta(\alpha, \lambda)} \in \mathbb{N}
$$

there exists a non-trivial solution of $(P)_{\lambda}$.

The next Lemma will give us information about the behavior of the function $\theta(\cdot, \lambda)$ for all $\lambda>0$. Since the proof uses the same ideas of [4], it is omitted.

Lemma 2.1. Let $\varphi$ satisfy conditions $\left(A_{0}\right),\left(A_{\infty}\right)$ and $f$ satisfy conditions $(1.1),(1.2)$. Then for all $\lambda>0$ the function $\theta(\cdot, \lambda)$ is continuous in $\mathbb{R}^{+}$and verify;

(i) $\lim _{\alpha \rightarrow+\infty} \theta(\alpha, \lambda)=0$ for $A=+\infty$

(ii) $\lim _{\alpha \rightarrow 0} \theta(\alpha, \lambda)=0$ for $\quad a=+\infty$

(iii) $\lim _{\alpha \rightarrow+\infty} \theta(\alpha, \lambda)=+\infty$ for $A=0$

(iv) $\lim _{\alpha \rightarrow 0} \theta(\alpha, \lambda)=+\infty$ for $\quad a=0$

(v) $\lim _{\alpha \rightarrow+\infty} \theta(\alpha, \lambda)=\frac{1}{2}\left(\frac{\lambda_{1}(p)}{\lambda A}\right)^{1 / p}$ for $0<A<+\infty$

(vi) $\lim _{\alpha \rightarrow 0} \theta(\alpha, \lambda)=\frac{1}{2}\left(\frac{\lambda_{1}(q)}{\lambda a}\right)^{1 / q}$ for $0<a<+\infty$.

In the next section we will write $\theta(+\infty, \lambda)$ and $\theta(0, \lambda)$, instead of $\lim _{\alpha \rightarrow+\infty} \theta(c$ and $\lim _{\alpha \rightarrow 0} \theta(\alpha, \lambda)$ respectively.

\section{Positive Solutions}

We now state and prove our main results.

Theorem 3.1. Suppose that $\varphi$ satisfy $\left(A_{0}\right),\left(A_{\infty}\right)$ and $f$ satisfy $(1.1),(1.2)$.

(i) If $\frac{\lambda_{1}(p)}{A}<\frac{\lambda_{1}(q)}{a}$ then problem $(P)_{\lambda}$ has at least one positive solution for any $\frac{\lambda_{1}(p)}{A}<\lambda<\frac{\lambda_{1}(q)}{a}$.

(ii) If $\frac{\lambda_{1}(q)}{a}<\frac{\lambda_{1}(p)}{A}$ then problem $(P)_{\lambda}$ has at least one positive solution for any $\frac{\lambda_{1}(q)}{a}<\lambda<\frac{\lambda_{1}(p)}{A}$. 
Proof. Let $\frac{\lambda_{1}(p)}{A}<\lambda<\frac{\lambda_{1}(q)}{a}$, it is inmediate that $\frac{1}{2}\left(\frac{\lambda_{1}(p)}{\lambda A}\right)^{1 / p}<$ $\frac{1}{2}<\frac{1}{2}\left(\frac{\lambda_{1}(q)}{\lambda a}\right)^{1 / q}$ then, by Lemma 2.1 we have $\theta(+\infty, \lambda)<\frac{1}{2}<\theta(0, \lambda)$. Since $\theta(\cdot, \lambda)$ is continuous the result of part (i) follows by application of the Intermediate Value Theorem. Part (ii) of the theorem can be proved in the same way.

Remark 3.1 Taking $A=+\infty$ (the superlinear case) one can prove that $(P)_{\lambda}$ has at least one positive solution for any $0<\lambda<\frac{\lambda_{1}(q)}{a}$. If in addition we take $a=0$, it also can be proved that $(P)_{\lambda}$ has at least one positive solution for all $\lambda>0$. Similar results are possible to obtain in the sublinear case, that is, when $a=+\infty$.

Theorem 3.2. Suppose that $\varphi$ satisfy $\left(A_{0}\right),\left(A_{\infty}\right)$ and $f$ satisfy $(1.1),(1.2)$. Assume that the function

$$
\psi_{t}(x)=\frac{F(t x)}{F(x)}
$$

is non-decreasing in $x>0$, for all $t \in(0,1)$.

(i) If $a=A=+\infty$ then there exists a constant $\lambda^{*}>0$ such that $(P)_{\lambda}$ has at least two positive solutions for any $0<\lambda<\lambda^{*}$, has at least one positive solution for $\lambda=\lambda^{*}$ and has no positive solutions for any $\lambda>\lambda^{*}$.

(ii) If $a=A=0$ then there exists a constant $\lambda_{*}>0$ such that $(P)_{\lambda}$ has no solutions for any $0<\lambda<\lambda_{*}$, has at least one positive solution for $\lambda=\lambda_{*}$ and has at least two positive solutions for any $\lambda>\lambda_{*}$.

Proof. The function $\theta(\alpha, \cdot)$ is decreasing in $(0,+\infty)$ for all $\alpha>0$. In fact, let $\lambda_{1}<\lambda_{2}$, using (2.3) we have $S\left(\alpha, \lambda_{1}\right)>S\left(\alpha, \lambda_{2}\right)$. Now, from (2.5) and $(2.3)$ it is clear that

$$
\theta(\alpha, \lambda)=\int_{0}^{1} \frac{S(\alpha, \lambda) d t}{W_{+}^{-1}\left(W(\alpha)\left(1-\frac{F(t S(\alpha, \lambda))}{F(S(\alpha, \lambda))}\right)\right.},
$$

and since $\psi_{t}(\cdot)$ is non-decreasing, the result is inmediate.

Let $a=A=+\infty$, by Lemma $2.1 \theta(0, \lambda)=\theta(+\infty, \lambda)=0$. We obscrve that from (3.2) we obtain

$$
\theta(\alpha, \lambda) \geq \frac{S(\alpha, \lambda)}{\alpha}
$$


thus, if we take $\alpha$ and $\lambda$ constants such that

$$
\frac{S(\alpha, \lambda)}{\alpha}>\frac{1}{2}
$$

there exist at least two positive solutions of $(P)_{\lambda}$.

On the other hand, (2.3) and (3.3) imply that

$$
\lambda<\frac{W(\alpha)}{F\left(\frac{\alpha}{2}\right)} .
$$

Thus, we define

$$
\mu^{*}=: \max \left\{\frac{W(\alpha)}{F\left(\frac{\alpha}{2}\right)} \mid \alpha>0\right\}
$$

to obtain that,

(i) For each $\lambda \in\left(0, \mu^{*}\right)$, the problem $(P)_{\lambda}$ has at least two positive solutions.

(ii) For $\lambda=\mu^{*}$, the problem $(P)_{\lambda}$ has at least one positive solution.

Moreover, if $(P)_{\lambda_{0}}$ has a positive solution for some $\lambda_{0}>0$, then $(P)_{\lambda}$ has a positive solution for each $\lambda \in\left(0, \lambda_{0}\right\}$, this is inmediate using the monotonicity of $\theta(\alpha, \cdot)$.

Now, we consider the following set

$$
S=\left\{\lambda>0 \mid(P)_{\eta} \text { has positive solution for all } \eta \leq \lambda\right\} .
$$

Clearly $S \neq \emptyset$ and is upper bounded, so we can define

$$
\lambda^{*}=\sup S .
$$

We claim that for any $0<\lambda<\lambda^{*}$ problem $(P)_{\lambda}$ has at least two positive solutions. In fact, from the definition of $\lambda^{*}$, and since $\theta(\alpha, \cdot)$ is decreasing, there exist $\lambda_{0} \in\left(\lambda, \lambda^{*}\right)$ and $\alpha_{\lambda_{0}} \in(0,+\infty)$ such that

$$
\frac{1}{2}=\theta\left(\alpha_{\lambda_{0}}, \lambda_{0}\right)<\theta\left(\alpha_{\lambda_{0}}, \lambda\right),
$$

from the continuity of $\theta(\cdot, \lambda)$ the result is inmediate. 
On the other hand, if $\lambda=\lambda^{*}$ we know that $(P)_{\lambda^{*}-\frac{1}{n}}$ ha $\mathrm{s}$ a positive solution for each $n \in \mathbb{N}$, then wca obtain a boued sequence $\left(\alpha_{n}\right)_{n \in \mathbb{N}} \subset$ $(0,+\infty)$ satisfying

$$
\theta\left(\alpha_{n}, \lambda^{*}-\frac{1}{n}\right)=\frac{1}{2} \quad \text { for all } n \in \mathbb{N} .
$$

Thus, there exists a convergent subsequence $\left(\alpha_{n_{k}}\right)_{k \in \mathbb{N}}$ such that

$$
\alpha_{n_{k}} \longrightarrow \alpha_{\lambda^{*}} \in(0,+\infty) \quad \text { when } \quad k \rightarrow+\infty,
$$

and thon, using the continuity of $\theta(\cdot, \cdot)$ we obtain

$$
\theta\left(\alpha_{\lambda^{*}}, \lambda^{*}\right)=\frac{1}{2}
$$

that is, $(P)_{\lambda}$ has at least one positive solution.

Finally for any $\lambda>\lambda^{*}$ problem $(P)_{\lambda}$ has no positive solutions. In fact, if we suppose that $(P)_{\lambda}$ has positive solution for $\lambda>\lambda^{*}$, then $\lambda \in S$, but from the definition of the suppremum $\lambda \leq \lambda^{*}$; which is a contradiction.

Thus, part (i) of theorem is proved.

Part (ii) of the theorem can be proved in the same way.

Remark 3.2 If we suppose that $f$ is homogeneous (i.c. $f(x)=|x|^{p-2} x$, $p>1$ ), then $\psi_{t}(x)=t^{p}$ is constant in $x>0$, thus condition (3.1) in Theorem 3.2 is also satisfied. thus ce0.3cm

Remark 3.3 We know from Theorom 3.2(i) that $(P)_{\lambda}$ has no positive solutions for $\lambda>\lambda^{*}$, however it is possible to prove that in this case it has infinite solutions.

\section{Examples}

In the following examples, let $r, p, q>1$.

Example 4.1 Let us set the functions

$$
\varphi(x)=\left(1+|x|^{r}\right)^{\frac{p}{r}-1}|x|^{r-2} x \text { and } f(x)=|x|^{q-2} x .
$$

Then, conditions $\left(A_{0}\right)$ and $\left(A_{\infty}\right)$ are satisficd since

$$
\lim _{x \rightarrow 0} \frac{\varphi(\sigma x)}{\varphi(x)}=\sigma^{r-1} \text { and } \lim _{x \rightarrow+\infty} \frac{\varphi(\sigma x)}{\varphi(x)}=\sigma^{p-1} .
$$


We consider the following problem

$$
\left\{\begin{array}{l}
\left.-\left(\left(1+\left|u^{\prime}\right|^{r}\right)\right)^{\frac{p}{r}-1}\left|u^{\prime}\right|^{r-2} u^{\prime}\right)^{\prime}=\lambda u^{q-1} \\
u(0)=u(1)=0 .
\end{array}\right.
$$

When $p<q<r$ we have

$$
\lim _{x \rightarrow 0} \frac{f(x)}{\varphi(x)}=+\infty=\lim _{x \rightarrow+\infty} \frac{f(x)}{\varphi(x)}
$$

(i.e. $a=A=+\infty$ ) then by Theorem $3.2(i)$ there exists $\lambda^{*}>0$ such that (4.1) has at least two positive solutions for any $0<\lambda<\lambda^{*}$, has at least one positive solution for $\lambda=\lambda^{*}$ and has no positive solutions for any $\lambda>\lambda^{*}$.

When $r<q<p$ we have

$$
\lim _{x \rightarrow 0} \frac{f(x)}{\varphi(x)}=0=\lim _{x \rightarrow+\infty} \frac{f(x)}{\varphi(x)}
$$

(i.e. $a=A=0$ ) then by Theorem 3.2(ii) there exists $\lambda_{*}>0$ such that (4.1) has no solutions for any $0<\lambda<\lambda_{*}$, has at least one positive solution for $\lambda=\lambda_{*}$ and has at least two positive solutions for any $\lambda>\lambda_{*}$.

We note that,

(1) When $q<r$ and $q<p$, or, $q>r$ and $q>p$ it can be proved that (4.1) has at least one positive solution for all $\lambda>0$.

(2) When $p=r$ we obtain the $p$-Laplacian operator, then for $q<p$ $(p<q)$ the function $\theta(\cdot, \lambda)$ is non-decreasing (resp. decreasing), in $(0,+\infty)$ therefore (4.1) has one positive solution for all $\lambda>0$.

(3) When $p=r=q$, we obtain from (4.1) the eigenvalue problem for the $p$-Laplacian, (see [5]).

Example 4.2 Let us set the functions

$$
\varphi(x)=|x|^{r-2} \log \left(1+|x|^{q-1}\right) x \quad \text { and } \quad f(x)=\frac{|x|^{q-2} x\left(q+p|x|^{q}\right)}{\left(1+|x|^{q}\right)^{2-\frac{p}{q}}} .
$$

Then, condition $\left(A_{0}\right)$ and $\left(A_{\infty}\right)$ are satisfied since

$$
\lim _{x \rightarrow 0} \frac{\varphi(\sigma x)}{\varphi(x)}=\sigma^{r+q-2} \text { and } \lim _{x \rightarrow+\infty} \frac{\varphi(\sigma x)}{\varphi(x)}=\sigma^{r-1} .
$$


Now, when $q>p$ the function

$$
\psi_{t}(x)=\frac{F(t x)}{F(x)}
$$

is non-decreasing in $x>0$ for all $t \in(0,1)$. In this case

$$
F(x)=\left(1+|x|^{q}\right)^{\frac{p}{q}-1}|x|^{q}
$$

Now, if we suposse that $p>r$, we obtain

$$
\lim _{x \rightarrow 0} \frac{f(x)}{\varphi(x)}=+\infty=\lim _{x \rightarrow+\infty} \frac{f(x)}{\varphi(x)}
$$

(i.e. $a=A=+\infty$ ), then by Theorem 3.2 (i) there exists $\lambda^{*}>0$ such that the problem

$$
\left\{\begin{array}{l}
-\left(\left|u^{\prime}\right|^{r-2} \log \left(1+\left|u^{\prime}\right|^{q-1}\right) u^{\prime}\right)^{\prime}=\lambda\left(\frac{|u|^{q-2} u\left(q+p|u|^{q}\right)}{\left(1+|u|^{q}\right)^{2-\frac{p}{q}}}\right) \\
u(0)=u(1)=0
\end{array}\right.
$$

has at least two positive solutions for any $0<\lambda<\lambda^{*}$, has at least one positive solution for $\lambda=\lambda^{*}$, and has no positive solutions for any $\lambda>\lambda^{*}$.

Example 4.3 Let us set the functions

$$
\varphi(x)=\left\{\begin{array}{cl}
\frac{x \log \left(1+|x|^{q-1}\right)}{|x| \log \left(1+|x|^{1-p}\right)} & , x \neq 0 \\
0 & , x=0
\end{array}\right.
$$

and

$$
f(x)=|x|^{r-1} \log \left(1+|x|^{r}\right) x .
$$

Then, conditions $\left(A_{0}\right)$ and $\left(A_{\infty}\right)$ are satisfied since

$$
\lim _{x \rightarrow 0} \frac{\varphi(\sigma x)}{\varphi(x)}=\sigma^{q} \text { and } \lim _{x \rightarrow+\infty} \frac{\varphi(\sigma x)}{\varphi(x)}=\sigma^{p-1} .
$$

When $2 r>q-1$ and $r>p-1$ we have that

$$
\lim _{x \rightarrow 0} \frac{f(x)}{\varphi(x)}=0 \text { and } \lim _{x \rightarrow+\infty} \frac{f(x)}{\varphi(x)}=+\infty,
$$


(i.e. $a=0$ and $A=+\infty$ ), then by Theorem 3.1 problem

$$
\left\{\begin{array}{l}
-\left(\frac{u^{\prime} \log \left(1+\left|u^{\prime}\right|^{q-1}\right)}{\left|u^{\prime}\right| \log \left(1+\left|u^{\prime}\right|^{1-p}\right)}\right)^{\prime}=\lambda|u|^{r-1} \log \left(1+|u|^{r}\right) u \\
u(0)=u(1)=0
\end{array}\right.
$$

has at least one positive solution for all $\lambda>0$.

Let observe us that if we suppose that $2 r>q-1$ and $r<p-1$ we obtain

$$
\lim _{x \rightarrow 0} \frac{f(x)}{\varphi(x)}=0=\lim _{x \rightarrow+\infty} \frac{f(x)}{\varphi(x)}
$$

(i.e. $a=A=0$ ). But in this case it is not difficult to prove that there exists $\mu_{*}>0$ such that for any $0<\lambda<\mu_{*}$ problem (4.3) has no solution. Herc $\mu_{*}$ is defined in a similar way as in (3.4).

\section{References}

[1] Brezis H. and Turner E.L, On a class of superlinear elliptic problems. Comm. P.D.E., 2, pp. 601-614, (1977).

[2] De Figueiredo D.G., Lions P.L. and Nussbaum R.D., A priori estimates and existence of positive solutions of semilinear elliptic equations. J. Math. Pures Appl., 61, pp. 41-63, (1982).

[3] Garcia-Huidobro M., Manasevich R. and Ubilla P., Existence of Positive Solutions for some Dirichlet Problems with an Asymptotically Homogeneous Operator. Elec. J. of Diff. Equat., 10, pp. 1-22, (1995).

[4] Garcia-Huidobro M. and Ubilla P., Multiplicity of solutions for a class of nonlinear second order equations. Nonlinear Analysis T.M.A., Vol. 28, N0 9, pp. 1509-1520, (1997).

[5] Guedda M. and Veron L., Bifurcation phenomena associated to the p-Laplace operator. Trans. Amer. Math. Soc., 310, pp. 419-431, (1988).

[6] Lions P.L., On the existence of positive solutions of semilinear Elliptic Equations. Siam Review, 24, pp. 441-467, (1982). 
[7] Narukawa K. and Suzuky T., Nonlinear Eigenvalue Problem for a Modified Capillary Surface Equation. Funkcialaj Ekvacioj, 37, pp. 81-100, (1994).

[8] Resnick S. I., Extreme Values, Regular Vuriation and Point Processes. Applied Probability, Vol 4, Springer Verlag, (1987).

[9] Seneta E., Regularly Varying functions. Lecture Notes in Mathematics, 508, Springer Verlag, (1976).

[10] Ubilla P., Multiplicity results for the 1-dimensional generalized pLaplacian. J. Math. Anal. and Appl., 190, pp. 611-623, (1995).

Received : May 1998.

Edson Arrázola

IMECC-UNICAMP

Caixa postal 6065

Campinas, SP 13081-970

Brasil

and

\section{Pedro Ubilla}

Departamento de Matemática

Universidad de Santiago de Chile

Casilla 307

Correo 2

Santiago

Chile 